\title{
Beyond brown: polyphenol oxidases as enzymes of plant specialized metabolism
}

\author{
Michael L. Sullivan* \\ U.S. Dairy Forage Research Center, Agricultural Research Service, United States Department of Agriculture, Madison, WI, USA
}

\author{
Edited by: \\ K. Judith Webb, Aberystwyth \\ University, UK \\ Reviewed by: \\ Norberto Daniel lusem, Universidad \\ de Buenos Aires, Argentina \\ Alan Lloyd, University of Texas at \\ Austin, USA \\ *Correspondence: \\ Michael L. Sullivan, U.S. Dairy Forage \\ Research Center, Agricultural \\ Research Service, United States \\ Department of Agriculture, 1925 \\ Linden Drive, Madison, WI 53706, \\ USA \\ e-mail: michael.sullivan@ars.usda.gov
}

Most cloned and/or characterized plant polyphenol oxidases (PPOs) have catechol oxidase activity (i.e., they oxidize o-diphenols to o-quinones) and are localized or predicted to be localized to plastids. As a class, they have broad substrate specificity and are associated with browning of produce and other plant materials. Because PPOs are often induced by wounding or pathogen attack, they are most generally believed to play important roles in plant defense responses. However, a few well-characterized PPOs appear to have very specific roles in the biosynthesis of specialized metabolites via both tyrosinase (monophenol oxidase) and catechol oxidase activities. Here we detail a few examples of these and explore the possibility that there may be many more "biosynthetic" PPOs.

Keywords: betalains, aurones, tyrosine metabolism, L-DOPA, tyramine, 8-8' linked lignans, specialized metabolism
Polyphenol oxidases (PPOs) are copper containing enzymes that are nearly ubiquitous among plants (Mayer, 2006). They have catechol oxidase activity (oxidation of $o$-diphenols to their corresponding o-quinones, EC 1.10.3.1) and many also have the ability to hydroxylate monophenols to $o$-diphenols (tyrosinase, EC 1.14.18.1; Marshall and Yoruk, 2003; Mayer, 2006). Throughout this review, the term tyrosinase will refer to the enzyme activity that oxidizes monophenols to $o$-diphenols. In the literature, the designation "PPO" sometimes includes laccases (EC 10.3.2). These copper containing enzymes are capable of oxidizing a wide range of aromatic compounds (including some utilized by PPO as defined above) and in plants are thought to have roles in radical coupling of monolignols to form lignin and flavanoid polymerization in the cell wall [see Mayer and Staples (2002) and the introduction of Turlapati et al. (2011) for reviews of laccase function in higher plants]. In the remainder of this review, laccases are not considered in the discussion of PPOs in plant specialized metabolism.

In plants, PPOs are perhaps best know for their role in post harvest browning: secondary reactions of PPO-generated $o$-quinones with cellular nucleophiles lead to the familiar discoloration of fresh produce and other plant materials (Vamos-Vigyazo, 1981). In some cases, these quinone reactions may be useful: for example in the so-called fermentation process in tea production (Subramanian et al., 1999) or in helping to preserve protein in forage crops (Lee et al., 2004; Sullivan and Hatfield, 2006). Generally, however, such browning reactions are thought of as a negative in food processing. Consequently, much research on PPO has been driven by this aspect of the enzyme. Because many PPOs are induced by wounding or pathogen attack, it has long been suggested that PPOs may play a role in defense responses. Indeed, it has been shown that PPO plays such a role in tomato (Thipyapong et al., 2004). Nonetheless, exactly what roles this nearly ubiquitous enzyme plays in normal plant growth and development are largely unknown. Because some PPOs have tyrosinase (hydroxylation of a monophenol to an $o$-diphenol) activity, it had long been suggested that PPO was responsible for the production of caffeic acid from $p$-coumaric acid (see for example Vaughan and Butt, 1969). It now seems likely that most plants actually use a cytochrome P450 enzyme for this conversion in vivo (Schoch et al., 2001; Franke et al., 2002). Still, the enzymatic properties of PPOs are potentially capable of providing important functions in plant specialized metabolism. Here we present a few cases where tyrosinase and/or catechol oxidase activities of specific PPOs have been proposed or demonstrated to have a crucial role in some aspect of plant specialized metabolism. Are these cases exceptional, or the tip of the iceberg?

\section{BETALAIN BIOSYNTHESIS}

There are several steps in betalain biosynthesis that might utilize either the tyrosinase and catechol oxidase activities of PPO (see Gandia-Herrero and Garcia-Carmona, 2013 for a detailed review). The first step in betalain biosynthesis is conversion of tyrosine to L-DOPA (L-3,4-dihydroxyphenylalanine; Figure 1A). The resulting L-DOPA can be a substrate for DOPA 4,5-dioxygenase (DODA) that cleaves DOPA's aromatic ring to form 4,5-secoDOPA. The cleavage product spontaneously rearranges to form betalamic acid, which can condense with amino acids or other amine groups to form yellow betaxanthins. Condensation of betalamic acid with cyclo-DOPA forms the red betacyanin pigments. The catechol oxidase activity of PPO could be involved in the oxidation of DOPA to DOPA quinone that can spontaneously rearrange to form the cyclo-DOPA moiety of the red betacyanin betalains (see Gandia-Herrero and Garcia-Carmona, 2013 and references therein). However, recently a cytochrome P450, CYP76AD1, has been identified in beet via a bioinformatic 


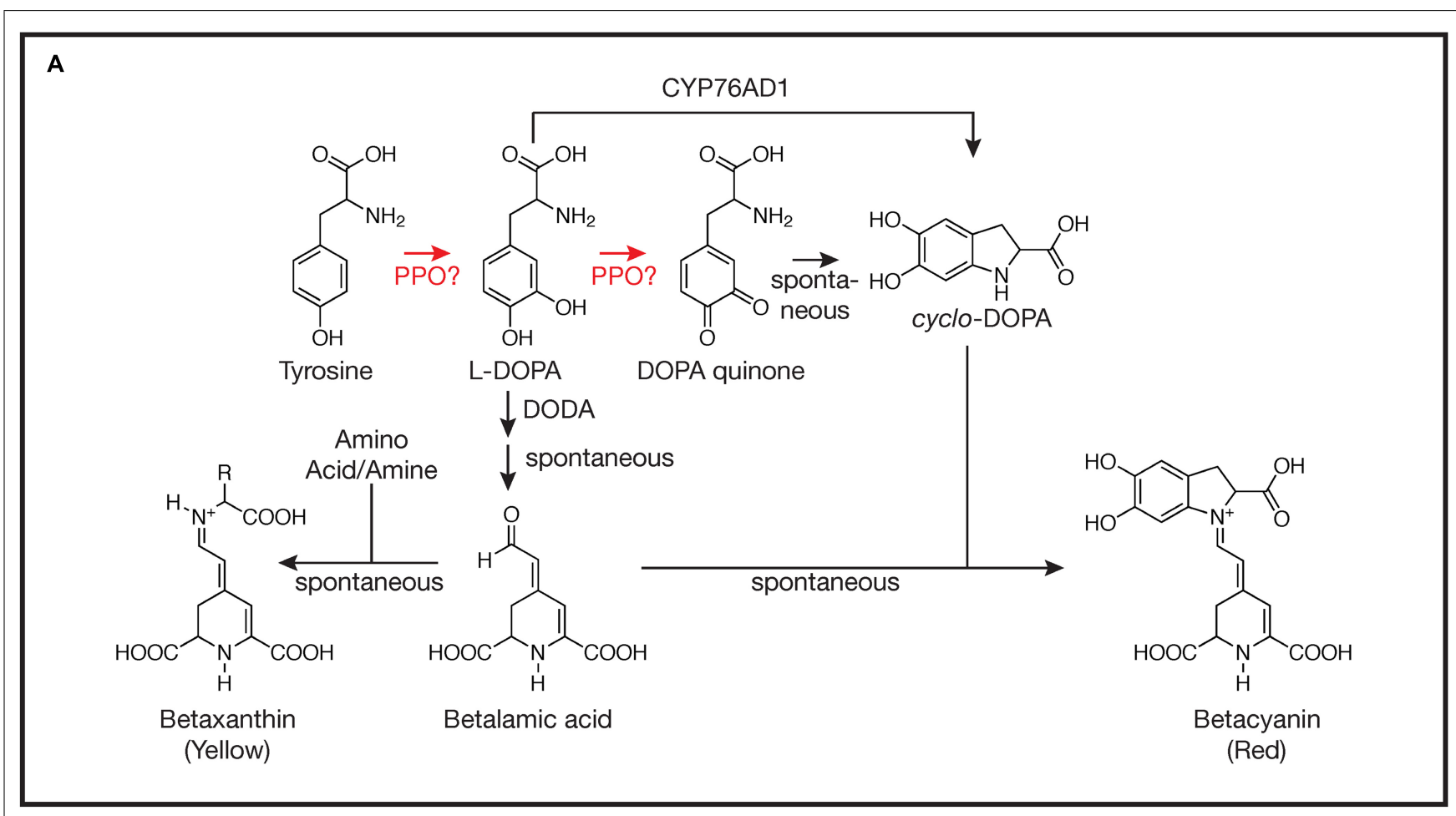

B

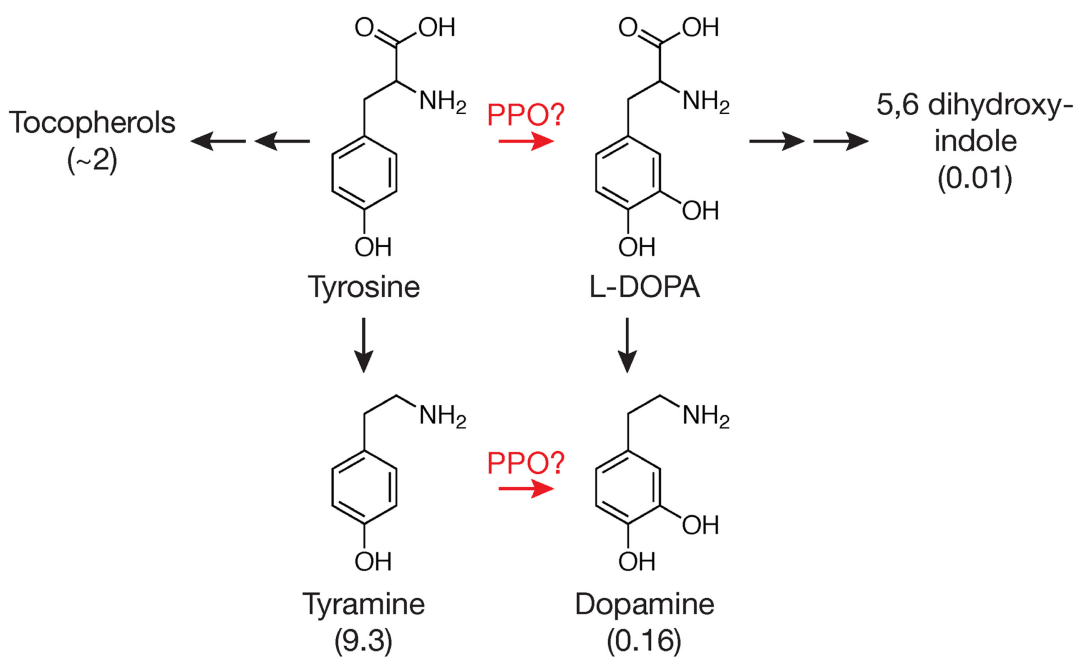

FIGURE 1 | Involvement of polyphenol oxidases (PPOs) in plant specialized metabolism. Steps where PPO involvement has been demonstrated or proposed are highlighted in red. For simplicity, not all reactants, enzymes, or stereochemistry are shown. Steps that occur spontaneously (not mediated by an enzyme) are indicated. (A) Betalain synthesis as described by Hatlestad etal. (2012), Gandia-Herrero and Garcia-Carmona (2013), and others. DODA, DOPA 4,5-dioxygenase; CYP76AD1, the cytochrome P450 described by
Hatlestad etal. (2012). The reaction mediated by CYP76AD1 likely proceeds via a DOPA quinone intermediate (Gandia-Herrero and Garcia-Carmona, 2013). (B) Proposed tyrosine metabolism in walnut (Araji etal., 2014). Values in parentheses are fold change in metabolite in walnut plants with PPO silenced via RNAi relative to wild type control plants as reported by Araji etal. (2014). Tyrosine levels did not change and L-DOPA itself was not detectable.

(Continued) 
C<smiles>CC1C(c2ccc(O)cc2)O[C@@H](c2ccc(O)cc2)C1C</smiles>

Larreatricin<smiles>CC1C(c2ccc(O)cc2)O[C@H](c2ccc(O)c(O)c2)C1C</smiles>

3'-Hydroxylarreatricin<smiles>CC1C(c2ccc(O)cc2)O[C@@H](c2ccc(O)c(O)c2)C1C</smiles><smiles>CC1C(c2ccc(O)c(O)c2)O[C@@H](c2ccc(O)c(O)c2)C1C</smiles>

3,3'-Dihydroxylarreatricin

D

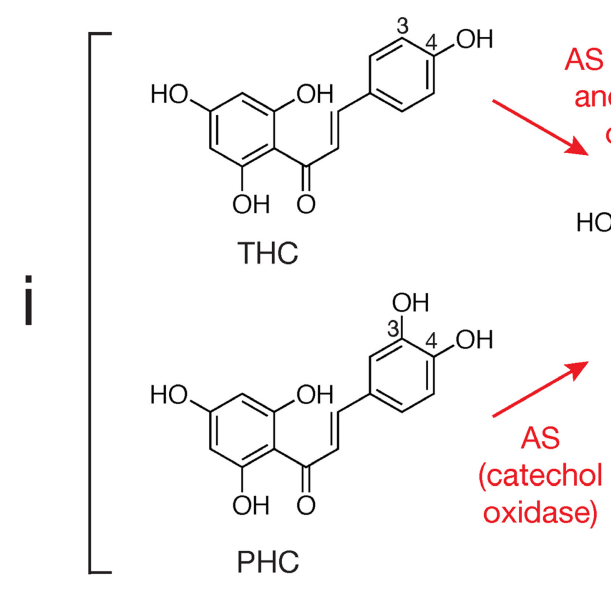

ii<smiles>O=C(/C=C/c1cccc(O)c1)c1ccc(O)cc1O</smiles>

Butein<smiles>O=C(O)O[AsH2]CO</smiles><smiles>O=C1C=CC(C=CC(=O)c2ccc(O)cc2O)=CC1=O</smiles>

Butein-quinone<smiles></smiles><smiles>O=C1/C(=C/c2ccc(O)c(O)c2)Oc2cc(O)ccc21</smiles>

Sulfuretin

\section{FIGURE 1 | Continued}

(C) 3,3'-Dihydroxylarreatricin biosynthesis in creosote bush as proposed by Cho etal. (2003). L3' H, larreatricin $3^{\prime}$-hydroxylase.

(D) (i) Aureusidin biosynthesis in $A$. majus as proposed by Nakayama etal. (2001). AS, aureusidin synthase (here, a vacuolar
PPO): THC, 2', 4', 6', 4-tetrahydroxychalcone; PHC, 2', 4', 6',3,4pentahydroxychalcone. For simplicity, bracteatin formation from $\mathrm{PHC}$ by AS is not shown. (ii) Sulfuretin biosynthesis in C. grandiflora as proposed by Kaintz etal. (2014). AS, aurone synthase (here, a plastidic PPO). 
approach that appears to carry out this reaction in vivo and can compliment the $\mathrm{R}$ mutant (produces yellow, but not red pigment) in beet and other species (Hatlestad et al., 2012). It has been suggested that CYP76AD1 could also be responsible for the initial tyrosine to L-DOPA oxidation as well. If this is the case in beet, however, the activity would be redundant with another, since silencing of CYP76AD1 results in loss of red, but not yellow pigments whose formation do not require cycloDOPA formation (Hatlestad et al., 2012). For the most part, the tyrosinase activity of PPO has been presumed to be the enzyme that mediates the initial tyrosine to L-DOPA conversion. Many studies show a correlation between tyrosinase enzyme activity and/or PPO gene expression and betalain pigment formation (see Steiner et al., 1999; Chang-Quan et al., 2007; Gao et al., 2009, for example). Further, a betaxanthin pathway can be recreated in tobacco cells using fungal PPO to carry out the tyrosine to L-DOPA step (Nakatsuka et al., 2013). However, transcriptome analysis in beet did not find the abundance of PPO transcripts that might be expected for high betalain production (Hatlestad et al., 2012), nor to our knowledge have PPO gene silencing experiments demonstrated a role for PPO in vivo. Thus, despite longstanding speculation that $\mathrm{PPO}$ is involved in betalain biosynthesis, its role in cyclo-DOPA formation seems unlikely, and definitive demonstration of a role in the initial conversion of tyrosine to L-DOPA in vivo is lacking.

\section{TYROSINE METABOLISM IN WALNUT}

Although a role for PPO in L-DOPA formation in betalain biosynthesis is far from clear, work by Araji et al. (2014) in walnut does support PPO-mediated conversion of tyrosine to L-DOPA, at least in some species. In walnut (Juglans regia), PPO is encoded by a single gene and has been demonstrated to have both tyrosinase and catechol oxidase activity (Escobar et al., 2008). To examine the in vivo function of PPO in walnut, Araji et al. (2014) created several RNAi transgenic lines that showed $>95 \%$ reductions in catechol oxidase activity relative to wild type controls. When placed in soil, these plants had a striking phenotype: they developed disease-like necrotic lesions. Despite the lesions, no pathogens could be identified from the leaves. Levels of salicylic acid, $\mathrm{H}_{2} \mathrm{O}_{2}$, or malondialdehyde (an indicator of oxidative damage), previously associated with other lesion-mimic mutants (Lorrain et al., 2003), were not significantly different in the PPO-silenced leaves compared to those of wild type leaves. Metabolomic analysis of $\mathrm{PPO}$-silenced and wild type leaves did reveal significant differences in many metabolites, however, particularly phenylpropanoids. Especially striking were changes in levels of compounds associated with tyrosine metabolism (Figure 1B; Araji et al., 2014). Compared to wild type leaves, those from PPO-silenced plants, had massively increased levels of tyramine (nearly 10 -fold), a primary metabolite of tyrosine, and substantial increases in tocopherols ( $\sim$ twofold), secondary metabolites of tyrosine. Conversely, levels of metabolites that would be expected to be derived from the 3-hydroxylation of tyrosine or tyramine (both good substrates for the tyrosinase activity of walnut PPO in vitro) were markedly reduced in PPO-silenced plants. Although L-DOPA was undetectable in both PPO-silenced and wild type walnut plants, levels of dopamine (derived from either L-DOPA or tyramine) and 5,6 dihydroxyindole (derived from L-DOPA) were reduced approximately 6- and 100-fold, respectively, in PPO-silenced plants relative to wild type controls (Figure 1B). Because the enzyme involved in 3-hydroxylation of these compounds had not been previously identified, the authors proposed that the simplest interpretation of the metabolomic results is that walnut PPO is the enzyme that mediates 3-hydroxylation of tyrosine and tyramine (Araji et al., 2014). Thus, silencing of PPO would be expected to result in increased accumulation of those tyrosine metabolites that do not undergo 3-hydroxylation such as tyramine and the tocopherols and decreased accumulation of metabolites derived from L-DOPA or tyramine. Further, the authors were able to demonstrate that the necrotic lesion phenotype of the PPOsilenced plants was almost certainly due to the accumulation of tyramine: incubation of petioles of detached wild type leaves in tyramine solution could phenocopy the necrotic lesions (Araji et al., 2014). Another metabolite that was dramatically decreased in PPO-silenced plants was esculetin. Although biosynthesis of this compound is not well understood, this observation is consistent with previous suggestion of the involvement of a chloroplast localized phenolase (Sato, 1967). More definitive demonstration of a central role of walnut PPO in tyrosine metabolism and esculetin biosynthesis in walnut might require approaches such as radioactive pulse labeling. It will be interesting to see how widespread this role of PPO in tyrosine metabolism is, especially in species whose PPO enzymes have been shown to have tyrosinase activity.

\section{BIOSYNTHESIS OF 8-8' LINKED LIGNANS IN CREOSOTE BUSH}

Another likely case of the tyrosinase activity of a PPO being involved in biosynthesis of a specialized metabolite is in the formation of $8-8^{\prime}$ linked lignans in creosote bush (Larrea tridentata). Many of these compounds from creosote bush, e.g., nordihydroguaiaretic acid (NDGA), have a number of bioactive properties including antiviral (Craigo et al., 2000), anticancer (McDonald et al., 2001) and allelopathtic properties (Elakovich and Stevens, 1985). Cho etal. (2003) used a combination of radiolabeled precursor experiments and metabolite identification to investigate the pathway of formation of NDGA and related compounds. Focusing on one of the steps, they postulated aromatic ring hydroxylation of larreatricin to form $3^{\prime}$ or 3-dihydroxylarreatricin (Figure 1C). A protein preparation from creosote bush was found to be capable of this activity, forming the $3^{\prime}$ - and 3-hydroxylarreatricin compounds in a ratio that favored the $3^{\prime}$ compound by $\sim$ seven fold. The larreatricin $3^{\prime}$ hydroxylase activity was purified to apparent homogeneity and showed enantiospecific hydroxylation, converting $(+)$-larreatricin to the corresponding $(+)-3^{\prime}$-hydroxylarreatricin, but not the $(-)$-enatomer. Peptide sequencing of the purified hydroxylase identified fragments with high homology to conserved domains of PPOs from other plant species. The peptide sequence data further allowed cloning and sequencing of a full length cDNA corresponding to the L. tridentata PPO. Like most plant PPOs, the L. tridentata PPO contains N-terminal sequences that would predict its localization to the chloroplast thylakoid lumen suggesting at least some of the steps of the synthesis of NDGA and related compounds take place in plastids. Unfortunately, in 
this study, reverse genetics (e.g., a gene silencing experiment) was not (or could not) be done nor was a recombinant protein product (e.g., produced in Arabidopsis, which lacks an endogenous PPO) characterized to more conclusively demonstrate the L. tridentate PPO gene product acts as the (+)-larreatricin $3^{\prime}$ hydroxylase in vivo. Nonetheless, the apparent homogeneity of the (+)-larreatricin $3^{\prime}$-hydroxylase purified from L. tridentate and its enantio-specificity for $(+)$-larreatricin are compelling. Unfortunately, there appears to have been relatively little further work on this pathway or characterization of the L. tridentate PPO. It would be interesting to know, for example, whether this same PPO mediates a second hydroxylation to form 3,3'-dihydroxylarreatricin (Figure 1C), and if not, what enzyme carries out that reaction. Does this PPO also form quinones from hydroxylarreatricins and under what conditions? Would such activities have any biological implications?

\section{AURONE BIOSYNTHESIS}

One of the most interesting and well-studied cases of PPO having a role in biosynthesis of specialized metabolites is the biosynthesis of the chalcone-derived yellow aurone pigments in snapdragon (Antirrhinum majus) flowers. It had been found that aurones (aureusidin and bracteatin) were formed from $2^{\prime}, 4^{\prime}, 6^{\prime}, 4$-tetrahydroxychalcone (THC) or $2^{\prime}, 4^{\prime}, 6^{\prime}, 3,4$ pentahydroxychalcone (PHC) upon incubation with extracts of yellow snapdragon flowers (Sato et al., 2001). The enzyme responsible, aureusidin (or aurone) synthase (AS), was purified to homogeneity from yellow snapdragon buds (Nakayama etal., 2000). Peptide sequencing of the purified enzyme allowed isolation and characterization of a cDNA encoding the enzyme. The predicted protein sequence showed high homology to other plant PPO enzymes. Expression of the gene corresponded to aurone accumulation (e.g., it was expressed in yellow flowers, but not in white or red flowers, nor in leaves) and expression developmentally coincided with levels of AS activity. Further, in vitro, tyrosinase from Neurospora crassa could also convert THC to aureusidin, indicating that the enzymatic activities of PPO are involved in the biosynthetic conversion. Subsequent detailed studies of AS substrate specificity allowed elucidation of a likely mechanism of aurone formation from THC or PHC involving both tyrosinase and catechol oxidase activities of the AS PPO (Figure 1Di; Nakayama et al., 2001). Starting with THC, tyrosinase and catechol oxidase activity result in 3-hydroxylation and formation of the corresponding o-quinone. Whether AS PPO carries out the 3hydroxylation reaction in vivo, or whether a cytochrome $\mathrm{P} 450$ chalcone 3-hydroxylase (as described below for Coreopsis grandiflora) is also involved has not been definitively established. AS likely forms the same quinone from PHC without the need for the 3hydroxylation step. The resulting quinone is predicted to undergo a 2-step non-enzyme mediated rearrangement to form aureusidin (Nakayama et al., 2001). Although the major product formed from PHC by AS is the $3^{\prime}, 4^{\prime}$-hydroxylated aureusidin, smaller amounts (approximately $1 / 6$ as much) of the $3^{\prime}, 4^{\prime}, 5^{\prime}$-hydroxylated bracteatin are also formed (not shown in Figure 1Di), suggesting AS is capable of adding a 5-hydroxyl to the PHC substrate. The $5^{\prime}$-hydroxylation of aureusidin by AS can be ruled out as the mechanism of bracteatin formation since incubation of aureusidin with AS failed to produce the product. AS was also unable to oxidize aureusidin to its corresponding quinone, nor could it oxidize several other mono and $o$-diphenolic compounds, such as tyrosine, p-coumaric acid, L-DOPA, caffeic acid, or eriodictyol, suggesting a relatively strict substrate specificity (Nakayama et al., 2001). One of the most novel aspects of the A. majus AS PPO is that it lacks the usual chloroplast targeting information that is common to most characterized PPOs (Nakayama et al., 2000). Subsequent studies using density gradient fractionation and GFPfusions with AS sequences demonstrated that AS is localized to vacuoles, where $4^{\prime}$-glucosides of the chalcone substrates, which may be the native substrates for AS, are also localized (Ono et al., 2006b). Consistent with this, Ono et al. (2006a) were able to produce aurones in flowers normally lacking them by expressing both the A. majus AS gene and a gene encoding chalcone $4^{\prime}$ $O$-glucosyltransferase from $A$. majus. Both genes were required, leading to the conclusion that chalcones are $4^{\prime}$-glycosylated in the cytoplasm leading to their transport to the vacuole where they can serve as substrates for A. majus AS. Recently, Kaintz et al. (2014) identified a PPO from C. grandiflora whose expression pattern is consistent with it being responsible for 4-deoxyaurone formation in this species (Figure 1Dii). Interestingly, the C. grandiflora AS PPO has relatively low sequence identity with $A$. majus AS PPO, is predicted to be plastid localized, and appears to lack tyrosinase activity. Consistent with the lack of tyrosinase activity, the C. grandiflora AS cannot utilize 4-monophenolic chalcone substrates and it seems likely that a cytochrome P450 chalcone 3-hydroxylase produces the 3,4-dihydroxy chalcones utilized by this AS (Schlangen etal., 2010; Kaintz et al., 2014). The plastidic versus vacuolar nature of the $C$. grandiflora and A. majus AS enzymes, respectively, indicate differences in the cell biology of aurone formation in these two systems, despite sharing some underlying PPO-mediated-chemistry.

\section{CONCLUDING REMARKS}

The above examples could represent the tip of the iceberg with respect to $\mathrm{PPO}$ enzymes that have specific roles in biosynthesis of specialized metabolites. Much work on PPOs has focused on their negative impact on food quality due to the browning reactions they promote. It could be that most of these characterized "food quality" PPOs are involved in general defense responses, leaving the impression that most PPOs are not particularly specialized. In two of the cases above, the specialized roles of the PPOs were identified in the course of research focused on a particular aspect of specialized metabolism. There, relatively laborious approaches led to the identification of the PPOs involved. For example, for both larreatricin 3'-hydroxylase (Cho et al., 2003) and A. majus AS (Nakayama et al., 2000), multistep protein purifications utilizing large amounts of plant tissue identified these PPO enzymes (for AS, $90 \mu \mathrm{g}$ of enzyme was purified from $32 \mathrm{~kg}$ of snapdragon buds!). Bioinformatics approaches will almost certainly facilitate identifying these specialized PPOs in the future. For example, in an analysis of PPO gene families from land plants whose genomes had been sequenced, Tran et al. (2012) identified 17 out of 83 PPO genes which lacked a chloroplast targeting signal and instead had either secretory pathway or unknown intracellular targeting. Although some of these could certainly 
be involved in defensive responses, such as seed defense (Anderson et al., 2010; Fuerst et al., 2014), it is intriguing to think that some represent PPOs involved in very specific processes including specialized metabolism, like the vacuolar targeted A. majus AS (Ono etal., 2006b). As they are becoming more routine, transcriptomic, metabolomic, and proteomic analyses could also provide useful information related to PPO function as was the case for walnut PPO discussed above. These types of analyses can provide answers to questions such as whether expression of a given PPO is tightly correlated with a phenotype of interest or whether a particular PPO is present in the subcellular compartment where a specific biosynthetic reaction is thought to occur. Thus genomics data, combined with other bioinformatic approaches, will almost certainly facilitate better understanding of PPO function in general, and the roles specific PPOs may play in specialized metabolism.

\section{ACKNOWLEDGMENTS}

We wish to thank Heather Green for helpful comments on the manuscript and Wayne Zeller for assistance in drawing chemical structures.

\section{REFERENCES}

Anderson, J. V., Fuerst, E. P., Tedrow, T., Hulke, B., and Kennedy, A. C. (2010). Activation of polyphenol oxidase in dormant wild oat caryopses by a seed-decay isolate of Fusarium avenaceum. J. Agric. Food Chem. 58, 10597-10605. doi: $10.1021 / \mathrm{jf} 102625 \mathrm{a}$

Araji, S., Grammer, T. A., Gertzen, R., Anderson, S. D., Mikulic-Petkovsek, M., Veberic, R., et al. (2014). Novel roles for the polyphenol oxidase enzyme in secondary metabolism and the regulation of cell death in walnut. Plant Physiol. 164, 1191-1203. doi: 10.1104/pp.113.228593

Chang-Quan, W., Heng, S., Xiang-Zhong, G., Qin-Guang, H., Feng, L., and BaoShan, W. (2007). Correlation of tyrosinase activity and betacyanin biosynthesis induced by dark in C-3 halophyte Suaeda salsa seedlings. Plant Sci. 173, 487-494. doi: 10.1016/j.plantsci.2007.07.010

Cho, M. H., Moinuddin, S. G. A., Helms, G. L., Hishiyama, S., Eichinger, D., Davin, L. B., et al. (2003). (+)-Larreatricin hydroxylase, an enantio-specific polyphenol oxidase from the creosote bush (Larrea tridentata). Proc. Natl. Acad. Sci. U.S.A. 100, 10641-10646. doi: 10.1073/pnas.1934562100

Craigo, J., Callahan, M., Huang, R. C. C., and Delucia, A. L. (2000). Inhibition of human papillomavirus type 16 gene expression by nordihydroguaiaretic acid plant lignan derivatives. Antiviral Res. 47, 19-28. doi: 10.1016/S01663542(00)00089-9

Elakovich, S. D., and Stevens, K. L. (1985). Phytotoxic properties of nordihydroguaiaretic acid, a lignan from Larrea tridentata (creosote bush). J. Chem. Ecol. 11, 27-33. doi: 10.1007/BF00987601

Escobar, M. A., Shilling, A., Higgins, P., Uratsu, S. L., and Dandekar, A. M. (2008). Characterization of polyphenol oxidase from walnut. J. Am. Soc. Hortic. Sci. 133, 852-858.

Franke, R., Humphreys, J. M., Hemm, M. R., Denault, J. W., Ruegger, M. O., Cusumano, J. C., et al. (2002). The Arabidopsis REF8 gene encodes the 3-hydroxylase of phenylpropanoid metabolism. Plant J. 30, 33-45. doi: 10.1046/j.1365-313X.2002.01266.x

Fuerst, E. P., Okubara, P. A., Anderson, J. V., and Morris, C. F. (2014). Polyphenol oxidase as a biochemical seed defense mechanism. Front. Plant Sci. 5:689. doi: $10.3389 /$ fpls.2014.00689

Gandia-Herrero, F., and Garcia-Carmona, F. (2013). Biosynthesis of betalains: yellow and violet plant pigments. Trends Plant Sci. 18, 334-343. doi: 10.1016/j.tplants.2013.01.003

Gao, Z. J., Han, X. H., and Xiao, X. G. (2009). Purification and characterisation of polyphenol oxidase from red Swiss chard (Beta vulgaris subspecies cicla) leaves. Food Chem. 117, 342-348. doi: 10.1016/j.foodchem.2009.04.013

Hatlestad, G. J., Sunnadeniya, R. M., Akhavan, N. A., Gonzalez, A., Goldman, I. L., Mcgrath, J. M., et al. (2012). The beet R locus encodes a new cytochrome
P450 required for red betalain production. Nat. Genet. 44, 816-820. doi: 10.1038/ng.2297

Kaintz, C., Molitor, C., Thill, J., Kampatsikas, I., Michael, C., Halbwirth, H., et al. (2014). Cloning and functional expression in E. coli of a polyphenol oxidase transcript from Coreopsis grandiflora involved in aurone formation. FEBS Let. 588, 3417-3426. doi: 10.1016/j.febslet.2014.07.034

Lee, M. R. F., Winters, A. L., Scollan, N. D., Dewhurst, R. J., Theodorou, M. K., and Minchin, F. R. (2004). Plant-mediated lipolysis and proteolysis in red clover with different polyphenol oxidase activities. J. Sci. Food Agric. 84, 1639-1645. doi: $10.1002 /$ jsfa. 1854

Lorrain, S., Vailleau, F., Balaque, C., and Roby, D. (2003). Lesion mimic mutants: keys for deciphering cell death and defense pathways in plants? Trends Plant Sci. 8, 263-271. doi: 10.1016/S1360-1385(03)00108-0

Marshall, M. R., and Yoruk, R. (2003). Physicochemical properties and function of plant polyphenol oxidase: a review. J. Food Biochem. 27, 361-422. doi: 10.1111/j.1745-4514.2003.tb00289.x

Mayer, A. M. (2006). Polyphenol oxidases in plants and fungi: going places? a review. Phytochemistry 67, 2318-2331. doi: 10.1016/j.phytochem.2006. 08.006

Mayer, A. M., and Staples, R. C. (2002). Laccase: new functions for an old enzyme. Phytochemistry 60, 551-565. doi: 10.1016/S0031-9422(02)00171-1

McDonald, R. W., Bunjobpon, W., Liu, T., Fessler, S., Pardo, O. E., Freer, I. K. A., et al. (2001). Synthesis and anticancer activity of nordihydroguaiaretic acid (NDGA) and analogues. Anti-Cancer Drug Des. 16, 261-270.

Nakatsuka, T., Yamada, E., Takahashi, H., Imamura, T., Suzuki, M., Ozeki, Y., et al. (2013). Genetic engineering of yellow betalain pigments beyond the species barrier. Sci. Rep. 3:1070. doi: 10.1038/srep01970

Nakayama, T., Soto, T., Fukui, Y., Yonekura-Sakakibara, K., Hayashi, H., Tanaka, Y., et al. (2001). Specificity analysis and mechanism of aurone synthesis catalyzed by aurenusidin synthase, a polyphenol oxidase homolog responsible for flower coloration. FEBS Lett. 499, 107-111. doi: 10.1016/S0014-5793(01) 02529-7

Nakayama, T., Yonekura-Sakakibara, K., Sato, T., Kikuchi, S., Fukui, Y., Fukuchi-Mizutani, M., et al. (2000). Aureusidin synthase: a polyphenol oxidase homolog responsible for flower coloration. Science 290, 1163-1166. doi: $10.1126 /$ science.290.5494.1163

Ono, E., Fukuchi-Mizutani, M., Nakamura, N., Fukui, Y., Yonekura-Sakakibara, K., Yamaguchi, M., et al. (2006a). Yellow flowers generated by expression of the aurone biosynthetic pathway. Proc. Natl. Acad. Sci. U.S.A. 103, 11075-11080. doi: $10.1073 /$ pnas. 0604246103

Ono, E., Hatayama, M., Isono, Y., Sato, T., Watanabe, R., Yonekura-Sakakibara, K., et al. (2006b). Localization of a flavonoid biosynthetic polyphenol oxidase in vacuoles. Plant J. 45, 133-143. doi: 10.1111/j.1365-313X.2005.02625.x

Sato, M. (1967). Metabolism of phenolic substances by chloroplasts.3. Phenolase as an enzyme concerning formation of esculetin. Phytochemistry 6, 1363-1373.

Sato, T., Nakayama, T., Kikuchi, S., Fukui, Y., Yonekura-Sakakibara, K., Ueda, T., etal. (2001). Enzymatic formation of aurones in the extracts of yellow snapdragon flowers. Plant Sci. 160, 229-236. doi: 10.1016/S0168-9452(00) 00385-X

Schlangen, K., Miosic, S., Thill, J., and Halbwirth, H. (2010). Cloning, functional expression, and characterization of a chalcone 3-hydroxylase from Cosmos sulphureus. J. Exp. Bot. 61, 3451-3459. doi: 10.1093/jxb/erq169

Schoch, G., Goepfert, S., Morant, M., Hehn, A., Meyer, D., Ullmann, P., et al. (2001). CYP98A3 from Arabidopsis thaliana is a 3'-hydroxylase of phenolic esters, a missing link in the phenylpropanoid pathway. J. Biol. Chem. 276, 36566-36574. doi: 10.1074/jbc.M104047200

Steiner, U., Schliemann, W., Bohm, H., and Strack, D. (1999). Tyrosinase involved in betalain biosynthesis of higher plants. Planta 208, 114-124. doi: $10.1007 / \mathrm{s} 004250050541$

Subramanian, N., Venkatesh, P., Ganguli, S., and Sinkar, V. P. (1999). Role of polyphenol oxidase and peroxidase in the generation of black tea theaflavins. J. Agric. Food Chem. 47, 2571-2578. doi: 10.1021/jf981042y

Sullivan, M. L., and Hatfield, R. D. (2006). Polyphenol oxidase and o-diphenols inhibit postharvest proteolysis in red clover and alfalfa. Crop Sci. 46, 662-670. doi: 10.2135/cropsci2005.06-0132

Thipyapong, P., Hunt, M. D., and Steffens, J. C. (2004). Antisense downregulation of polyphenol oxidase results in enhanced disease susceptibility. Planta 220, 105117. doi: $10.1007 / \mathrm{s} 00425-004-1330-6$ 
Tran, L. T., Taylor, J. S., and Constabel, C. P. (2012). The polyphenol oxidase gene family in land plants: Lineage-specific duplication and expansion. BMC Genomics 13:395. doi: 10.1186/1471-2164-13-395

Turlapati, P. V., Kim, K. W., Davin, L. B., and Lewis, N. G. (2011). The laccase multigene family in Arabidopsis thaliana: towards addressing the mystery of their gene function(s). Planta 233, 439-470. doi: 10.1007/s00425-010-1298-3

Vamos-Vigyazo, L. (1981). Polyphenol oxidase and peroxidase in fruits and vegetables. CRC Crit. Rev. Food Sci. Nutr. 15, 49-127. doi: 10.1080/10408398109 527312

Vaughan, P. F. T., and Butt, V. S. (1969). Hydroxylation of p-coumaric acid by an enzyme from leaves of spinach beet (Beta Vulgaris L.). Biochem. J. 113, 109-115.

Conflict of Interest Statement: The author declares that the research was conducted in the absence of any commercial or financial relationships that could be construed as a potential conflict of interest. The Guest Associate Editor Dr. K. Judith Webb declares that, despite having collaborated with author Dr. Michael L. Sullivan, the review process was handled objectively and no conflict of interest exists.

Received: 10 November 2014; accepted: 17 December 2014; published online: 14 January 2015.

Citation: Sullivan ML (2015) Beyond brown: polyphenol oxidases as enzymes of plant specialized metabolism. Front. Plant Sci. 5:783. doi: 10.3389/fpls.2014.00783

This article was submitted to Plant Physiology, a section of the journal Frontiers in Plant Science.

Copyright (c) 2015 Sullivan. This is an open-access article distributed under the terms of the Creative Commons Attribution License (CC BY). The use, distribution or reproduction in other forums is permitted, provided the original author(s) or licensor are credited and that the original publication in this journal is cited, in accordance with accepted academic practice. No use, distribution or reproduction is permitted which does not comply with these terms. 